\title{
A selection of maximal elements under non-transitive indifferences
}

\author{
José Carlos R. Alcantud ${ }^{\mathrm{a}, *}$, Gianni Bosi ${ }^{\mathrm{b}}$, Magalì Zuanon ${ }^{\mathrm{c}}$ \\ ${ }^{a}$ Facultad de Economía y Empresa, Universidad de Salamanca, E 37008 Salamanca, Spain \\ ${ }^{\mathrm{b}}$ Facoltà di Economia, Università degli Studi di Trieste, Piazzale Europa 1, 34127 Trieste, Italy \\ ${ }^{\text {c } F a c o l t a ̀ ~ d i ~ E c o n o m i a, ~ U n i v e r s i t a ̀ ~ d e g l i ~ S t u d i ~ d i ~ B r e s c i a, ~ C o n t r a d a ~ S a n t a ~ C h i a r a ~ 50, ~} 25122$ Brescia, Italy
}

\section{A R T I C L E I N F O}

Article history:

Received 19 August 2009

Received in revised form

4 August 2010

Available online 13 October 2010

\section{Keywords:}

Maximal element

Selection of maximals

Acyclicity

Interval order

Semiorder

\begin{abstract}
A B S T R A C T
In this work we are concerned with maximality issues under intransitivity of the indifference. Our approach relies on the analysis of "undominated maximals" (cf., Peris \& Subiza, 2002). Provided that an agent's binary relation is acyclic, this is a selection of its maximal elements that can always be done when the set of alternatives is finite. In the case of semiorders, proceeding in this way is the same as using Luce's selected maximals.

We present a sufficient condition for the existence of undominated maximals for interval orders without any cardinality restriction. Its application to certain types of continuous semiorders is very intuitive and accommodates the well-known "sugar example" by Luce.
\end{abstract}

(C) 2010 Elsevier Inc. All rights reserved.

\section{Introduction}

Even though there are arguments to ensure the existence of maximal elements for binary relations in very general settings, this concept does not always explain the choice under non-transitive indifference well. Luce (1956) argued that in order to account for certain procedural aspects better, some "selection of maximals" helps the researcher. From his Introduction: “... a maximization principle is almost always employed which states in effect that a rational being will respond to any finite difference in utility, however small. It is, of course, false that people behave in this manner". This author proposed the concept of a semiorder as a way to deal with intransitive indifferences without giving up transitivity of the strict preference.

In this work we are concerned with maximality considerations under intransitivity of the indifference. It is based on the analysis of "undominated maximals", a concept introduced and explored by Peris and Subiza (2002). They established two particularly remarkable facts. For one thing, such selection of maximals can be done when the set of alternatives is finite provided that the binary relation is acyclic. Then, proceeding in this way is the same as using Luce's selected maximals in the case of semiorders. ${ }^{1}$

\footnotetext{
* Corresponding author.

E-mail address: jcr@usal.es (J.C.R. Alcantud).

URL: http://web.usal.es/jcr (J.C.R. Alcantud).

1 Luce (1956, Section 3) states that in terms of a semiorder on a set it is possible to define a natural weak ordering on the same set. Further, if the semiorder is a
}

In light of these two facts, it seems interesting to provide conditions for the existence of undominated maximals without any cardinality restriction. We present a condition that is sufficient for the existence of undominated maximal elements in interval orders. Its specialization to certain types of continuous semiorders is very intuitive and accommodates the well-known "sugar example" by Luce.

This work is organized as follows. In Section 2, we establish our notation and preliminary definitions. Then in Section 3, we analyse the existence of undominated maximals in the case of unrestricted cardinality of the set of alternatives. As an application, a concrete specification leading to Luce's analysis of the "sugar example" is provided. Finally, we investigate the role of different assumptions in our results. Section 4 contains some conclusions and remarks.

\section{Notation and preliminaries}

Let us fix a set $X$ of alternatives. Unless otherwise stated, henceforth $\succ$ denotes an acyclic relation, i.e., $x_{1} \succ x_{2} \succ \cdots \succ x_{n}$ implies $x_{1} \neq x_{n}$ for all $x_{1}, \ldots, x_{n} \in X$. Its lower (resp., upper) contour set associated with $x \in X$ is $\{z \in X: x>z\}$ (resp., $\{z \in X: z \succ x\}$ ). A subset $A \subseteq X$ is a lower (resp., upper) set of $\succ$ when $a \in A, x \in X$, and $a \succ \bar{x}$ (resp., $x \succ a$ ) implies $x \in A$.

weak order then the induced weak ordering is identical to the given one. The term Luce's selected maximals of a semiorder refers to the maximal elements of such weak ordering, provided that they exist. Example 2 formalizes this construction. 
Denote by $\succsim$ the complement of the dual of $\succ$ (i.e., $x \succsim y$ if and only if $y \succ x$ is false), and by $\sim$ the indifference relation associated with $\succ$ (i.e., $x \sim y$ if and only if both $x \succsim y$ and $y \succsim x$ ).

With every acyclic relation $\succ$ on $X$ we associate the traces $\succ^{*}$ and $\succ^{* *}$ defined as follows: for all $x, y \in X$,

$x \succ^{*} y \Leftrightarrow \exists \xi \in X: x \succ \xi \succsim y$,

$x \succ^{* *} y \Leftrightarrow \exists \eta \in X: x \succsim \eta \succ y$.

Therefore, if we denote by $\succsim^{*}$ and $\succsim^{* *}$ the respective complements of the duals of $\succ^{*}$ and $\succ^{* *}$, we have

$x \succsim^{*} y \Leftrightarrow(y \succ z \Rightarrow x \succ z)$,

$x \succsim^{* *} y \Leftrightarrow(z \succ x \Rightarrow z \succ y)$.

We recall that a binary relation $\succ$ on $X$ is an interval order if it is irreflexive and the following condition is verified for all $x, y, z, w \in$ $X$ :

$(x \succ z)$ and $(y \succ w) \Rightarrow$

$(x \succ w)$ or $(y \succ z)$.

Further, a binary relation $\succ$ on $X$ is a semiorder if $\succ$ is an interval order and the following condition is verified for all $x, y, z, w \in X$ :

$(x \succ y)$ and $(y \succ z) \Rightarrow$

$(x \succ w)$ or $(w \succ z)$.

If $\succ$ is an interval order then $\succ^{*}$ and $\succ^{* *}$ are weak orders (i.e., asymmetric and negatively transitive binary relations). The traces $\succ^{*}$ and $\succ^{* *}$ extend the interval order $\succ$ (i.e., $x \succ y$ implies both $x \succ^{*} y$ and $x \succ^{* *} y$ for all $x, y \in X$ ). If $\succ$ is a semiorder then the binary relation $\succ^{0}=\succ^{*} \cup \succ^{* *}$ is a weak order (cf., Fishburn, 1985, Theorem 2 of Section 2) and therefore we have that $x \succ^{*} y$ implies that $x \succsim^{* *} y$ for all $x, y \in X$.

Using the terminology of Peris and Subiza (2002), the weak dominance relation $\succsim^{D}$ and the strict dominance relation $\succ^{D}$ associated with an interval order $\succ$ on a set $X$ can be defined as follows: for each $x, y \in X$,

$x \succsim^{D} y \Leftrightarrow x \succsim^{*} y$ and $x \succsim^{* *} y$,

$x \succ^{D} y \Leftrightarrow x \succsim^{D} y$ and not $\left(y \succsim^{D} x\right)$.

We denote by $M(X, \succ)$ the set of maximal elements relative to $\succ$ on $X$, i.e., $M(X, \succ)=\{x \in X: \forall z \in X, z \succ x$ is false $\}$.

If $\tau$ is a topology on $X, \succ$ is upper semicontinuous if its lower contour sets are open. From Alcantud (2002), we say that $(X, \tau)$ is $\succ$-upper compact if for each collection of lower open sets that covers $X$ there exists a finite subcollection that also covers $X$.

\section{Selection of maximal elements for acyclic relations}

The set of undominated maximal elements of $X$ is defined as

$U M(X, \succ)=M(X, \succ) \cap M\left(X, \succ^{D}\right)$.

If we restrict ourselves to finite sets, undominated maximal elements do exist under acyclicity of $\succ$ (cf., Peris \& Subiza, 2002, Theorem 2). In Section 3.1 we show that even if we focus on semiorders and impose classical (and restrictive) conditions in the vein of the Bergstrom-Walker theorem, when $X$ is infinite the set of undominated maximals may be empty. Then in Section 3.2, we produce general conditions for the existence of undominated maximals on topological spaces with arbitrary cardinality. Section 3.3 yields a corollary with an application to a celebrated analysis by Luce.

\subsection{Undominated maximals vs. maximal elements}

Besides finiteness, the literature has provided many additional conditions under which maximal elements do exist. There are various approaches to that issue but the most celebrated one probably is the Bergstrom-Walker theorem and its variations (cf., Bergstrom, 1975; Walker, 1977). Its basic form states that upper semicontinuous acyclic relations on compact topological spaces have maximal elements. Example 1 shows that even in the case of semiorders on countable sets, this specification does not suffice to ensure that undominated maximals exist.

Example 1. Let us fix $A=\mathbb{N}=\{1,2,3, \ldots\}$. The next expression produces an upper semicontinuous semiorder with respect to the excluded point topology associated with $\{1\}$ on $A$, which is always compact $^{2}$ :

$m \succ n$ if and only if $m$ is odd, $n$ is even, and $m+1 \geqslant n$.

Although $A$ has an infinite number of maximal elements (namely, the odd numbers) there are no undominated maximals because $(m+2) \succ^{D} m$ when $m$ is odd.

If we adhere to the topological approach in our quest for conditions that guarantee that undominated maximals do exist, then we need to consider other suitable assumptions. That is the purpose of Section 3.2.

\subsection{Existence of undominated maximals for unrestricted domains}

The next lemma shows that an alternative expression for the set of undominated maximals can be given only under acyclicity of $\succ$.

Lemma 1. Suppose that $\succ$ is an acyclic relation on $X$. Then $U M(X, \succ)=M\left(M(X, \succ), \succ^{D}\right)$.

Proof. Along the proof of Peris and Subiza (2002, Theorem 2) the inclusion $M\left(M(X, \succ), \succ^{D}\right) \subseteq U M(X, \succ)$ is proved. The fact that every $x \in U M(X, \succ)$ belongs to $M\left(M(X, \succ), \succ^{D}\right)$ is immediate.

Next we present some technical and useful properties that hold in our setting.

Lemma 2. Suppose that $\succ$ is an acyclic relation on $X$. Then,

(1) $\succ^{D} \subseteq \succ^{*}$ on $M(X, \succ)$,

(2) $M\left(X, \succ^{*}\right) \subseteq M(X, \succ)$.

Proof. In order to check (1) we notice that the original expression for $\succ^{D}$, namely

$x \succ^{D} y \Leftrightarrow\left\{\begin{array}{l}x \succ^{*} y \text { and } x \succsim^{* *} y \\ \text { or } \\ x \succ^{* *} y \text { and } x \succsim^{*} y\end{array}\right.$

can be simplified because now $x \succ^{* *} y$ is impossible since $y$ is maximal for $\succ$. This fact rules out (b) and yields the conclusion.

Part (2) is direct because $\succ^{*}$ extends $\succ$ : if $x \succ y$ then $x \succ y \succsim y$ due to irreflexivity of $\succ$.

Remark 1. Besides Lemma 2(1), we can further note that if $\succ$ is a semiorder then $\succ^{D}=\succ^{*}$ on $M(X, \succ)$. Thus $x, y \in U M(X, \succ)$ $=M\left(M(X, \succ), \succ^{D}\right)=M\left(M(X, \succ), \succ^{*}\right)$ now yields $x \sim^{*} y$. Because $x \sim^{* *} y$ is trivial here, we conclude as in Peris and Subiza (2002, Proposition 2) that $x \approx y$ when $x, y \in U M(X, \succ)$ (but for semiorders only). ${ }^{3}$ 2.1).

The following result was proven by Bridges (1985, Proposition

Lemma 3. Suppose that $\succ$ is an irreflexive relation on $X$. Then $\succ^{*}$ is asymmetric if and only if $\succ$ is an interval order. ${ }^{4}$

\footnotetext{
2 The open sets are the subsets of $A$ that do not contain 1 plus $A$.

3 The reader can verify that $\approx=\sim^{*} \cap \sim^{* *}$ is Fishburn's equivalence relation as defined in Peris and Subiza (2002, page 3).

4 We do not use the fact that these conditions are also equivalent to the asymmetry of $\succ^{* *}$, which is stated in Bridges' proposition.
} 
We are ready to present our main result.

Theorem 1. Suppose that $\succ$ is an irreflexive relation on a topological space $X$. If $\succ^{*}$ is asymmetric and upper semicontinuous and $X$ is $\succ^{*}$ upper compact then $\succ$ has undominated maximal elements on $X$.

Proof. The relation $\succ$ must be an interval order by Lemma 3; therefore it is acyclic. Proposition 2 in Alcantud (2002) ensures that $M\left(X, \succ^{*}\right)$ is non-empty and $\succ^{*}$-upper compact. We can apply Lemma 2(2) to deduce $\varnothing \neq M\left(X, \succ^{*}\right) \subseteq M\left(M(X, \succ), \succ^{*}\right)$ : if $x \in M\left(X, \succ^{*}\right) \subseteq M(X, \succ)$ does not belong to $M\left(M(X, \succ), \succ^{*}\right)$ then there exists $y \in M(X, \succ)$ such that $y \succ^{*} x$, a contradiction. Now we use Lemma 2(1) to produce $\varnothing \neq M\left(M(X, \succ), \succ^{*}\right) \subseteq$ $M\left(M(X, \succ), \succ^{D}\right)$ and then Lemma 1 in order to enforce

$\varnothing \neq M\left(M(X, \succ), \succ^{D}\right)=U M(X, \succ)$.

\subsection{An application to Luce's maximal elements}

Corollary 1. Suppose that $\succ$ is a continuous interval order with respect to a given topology on $X$, and that $\succ^{*}=\succ^{* *}$. Then $X$ has undominated maximals as long as it is $\succ^{*}$-upper compact.

Proof. Because $\succ^{*} \cup \succ^{* *}=\succ^{*}=\succ^{* *}$ is a weak order, $\succ$ is a semiorder. Besides, $\succ^{*}=\succ^{* *}$ is continuous because $\succ^{*}$ is lower semicontinuous and $\succ^{* *}$ is upper semicontinuous (cf., Bosi, Candeal, Induráin, Oloriz, \& Zudaire, 2001, proof of implication (ii) $\Rightarrow$ (iii) in Theorem 3). Now Theorem 1 applies.

The following example provides a well-known application of Corollary 1.

Example 2. Consider the case where $X=\mathbb{R}$ with the usual topology, and take any $u: X \longrightarrow \mathbb{R}$ continuous and $K>0$. Then the continuous semiorder defined by $x>y$, if and only if $u(x)>$ $u(y)+K$, satisfies $\succ^{*}=\succ^{* *}$ (cf., Campión, Candeal, Induráin, \& Zudaire, 2008, Theorem 3.5). Therefore $\succ$ has undominated maximals on any compact set. In particular, let $u=\mathrm{i} d$ and $K=2$. It is immediate that $\succ^{*}=\succ^{* *}=\succ^{*} \cup \succ^{* *}$ is the usual order of the real numbers. Then the compact set $C=\{15,16,17,18,19,20\}$ has undominated maximal elements. Moreover they coincide with Luce's maximals (namely, $\operatorname{LM}(C, \succ)=M\left(C, \succ^{*} \cup \succ^{* *}\right)$ ) because for any semiorder $P$ on a set $A$ the equality $\operatorname{LM}(A, P)=U M(A, P)$ holds true by Peris and Subiza (2002, Theorem 4(c)). This is how Luce's maximal set selects $\{20\}$, the "true" maximal element in Luce's “sugar example” (cf., e.g., Peris \& Subiza, 2002, page 4).

We proceed to prove that the assumption that $\succ^{*}=\succ^{* *}$ in Corollary 1 is not superfluous. Example 4 shows that the same holds for the $\succ^{*}$-upper compactness assumption.

Example 3. Take the semiorder given by Example 1 but now endow the set $A$ with the topology specified by the following basis (cf., Munkres, 1975, Section 13 or Willard, 1970, Section 2.5):

$\{\{m, m+2, m+4, \ldots\}: m$ is odd $\} \bigcup\{\{2,4,6, \ldots, n\}: n$ is even $\}$.

Then $\succ$ is continuous and the topology is $\succ^{*}$-upper compact because $\succ^{*}$ is the weak order given by

$\cdots \succ^{*} 5 \succ^{*} 3 \succ^{*} 1$,

$2 \sim^{*} 4 \sim^{*} 6 \sim \ldots, 1 \succ^{*} 2$.

Thus the only lower (with respect to $\succ^{*}$ ) open set that contains 1 is $A=\mathbb{N}$. Also, because $\succ^{* *}$ is the weak order given by

$1 \sim^{* *} 3 \sim * * 5 \sim \ldots$,

$\cdots \succ^{* *} 6 \succ^{* *} 4 \succ^{* *} 2$,

$1 \succ^{* *} n$ for each even $n$

it is apparent that $\succ^{*} \neq \succ^{* *}$.

\subsection{On the assumptions of Theorem 1}

Theorem 1 shows that an adequate relationship between the binary relation and the topology on $X$ produces the desired conclusion. Examples 4 and 5 show that its assumptions (namely, upper semicontinuity and upper compactness of the topology with respect to $\succ^{*}$ ) are necessary for the existence of undominated maximal elements.

Example 4. Consider $B=[0,1) \subseteq \mathbb{R}$ in Example 2. Then its usual topology is not $\succ^{*}$-upper compact, and $\succ$ has not even maximal elements on $B$.

Therefore the $\succ^{*}$-upper compactness assumption is not superfluous in Theorem 1 . The same is true for Corollary 1.

Example 5. Consider $D=[0,+\infty) \subseteq \mathbb{R}$ in Example 2. If we endow it with the excluded point topology associated with 0 , then $\succ^{*}$ is not upper semicontinuous. Although $D$ is $\succ^{*}$-upper compact, $\succ$ has not even maximal elements on $D$.

Therefore upper semicontinuity of $\succ^{*}$ is not superfluous in Theorem 1.

\section{Concluding remarks}

In trying to fill the gap about lack of general conditions for the existence of certain selections of maximals, we have focused on at least acyclic relations because they are the natural setting for maximality purposes. Even in case that the relation is a semiorder, the usual conditions ensuring that maximal elements exist (namely, upper semicontinuity with respect to compact topologies) do not guarantee that undominated maximal elements exist.

The characterization of undominated maximality given by Lemma 1 seems to point at making assumptions on the strict dominance relation, because useful topological properties of the set of maximal elements are known (as recalled along the proof of Theorem 1).

We have explored an intuitive approach to this possibility, based on making assumptions on a trace of the original relation instead. ${ }^{5}$ Because the structure of interval orders is very rich and it is related to that of their traces, our proposal favours an especially intuitive specification for a case where the relation is in the class of semiorders (cf., Corollary 1 ). In light of Remark 1 we can ensure that assumptions on the strict dominance relation are being made in such cases. As a consequence, we deduce the existence of Luce's maximal elements in settings like the highly cited "sugar example".

\section{Acknowledgments}

The first author acknowledges financial support by the Spanish Ministerio de Ciencia e Innovación under Project ECO2009-07682, and by Junta de Castilla y León under Project SA024A08 and GR-99 Funding.

\section{References}

Alcantud, J. C. R. (2002). Characterization of the existence of maximal elements of acyclic relations. Economic Theory, 19, 407-416.

Bergstrom, T. C. (1975). Maximal elements of acyclic relations on compact sets. Journal of Economic Theory, 10, 403-404.

\footnotetext{
5 Our main theorem concerns at least interval orders, as Lemma 3 explains. Nonetheless its statement makes it clear that the assumptions that must be checked in order to apply it are very simple.
} 
Bosi, G., Candeal, J. C., Induráin, E., Oloriz, E., \& Zudaire, M. (2001). Numerical representations of interval orders. Order, 18, 171-190.

Bridges, D. S. (1985). Representing interval orders by a single real-valued function. Journal of Economic Theory, 36, 149-155.

Campión, M. J., Candeal, J. C., Induráin, E., \& Zudaire, M. (2008). Continuous representability of semiorders. Journal of Mathematical Psychology, 52, 48-54.

Fishburn, P. C. (1985). Interval orders and interval graphs. John Wiley \& Sons.
Luce, R. D. (1956). Semiorders and a theory of utility discrimination. Econometrica, 24, 178-191.

Munkres, J. (1975). Topology. Prentice Hall.

Peris, J. E., \& Subiza, B. (2002). Choosing among maximals. Journal of Mathematical Psychology, 46, 1-11.

Walker, M. (1977). On the existence of maximal elements. Journal of Economic Theory, 16, 470-474.

Willard, S. (1970). General topology. Addison-Wesley. 